\title{
REARWARD VISION, DRIVER CONFIDENCE, AND DISCOMFORT GLARE USING AN ELECTROCHROMIC REARVIEW MIRROR
}

\author{
Michael J. Flannagan \\ Michael Sivak \\ Andrew W. Gellatly
}

The University of Michigan

Transportation Research Institute

Ann Arbor, Michigan 48109-2150

U.S.A.

Report No. UMTRI-90-27

June 1990 
Technical Report Documentation Page

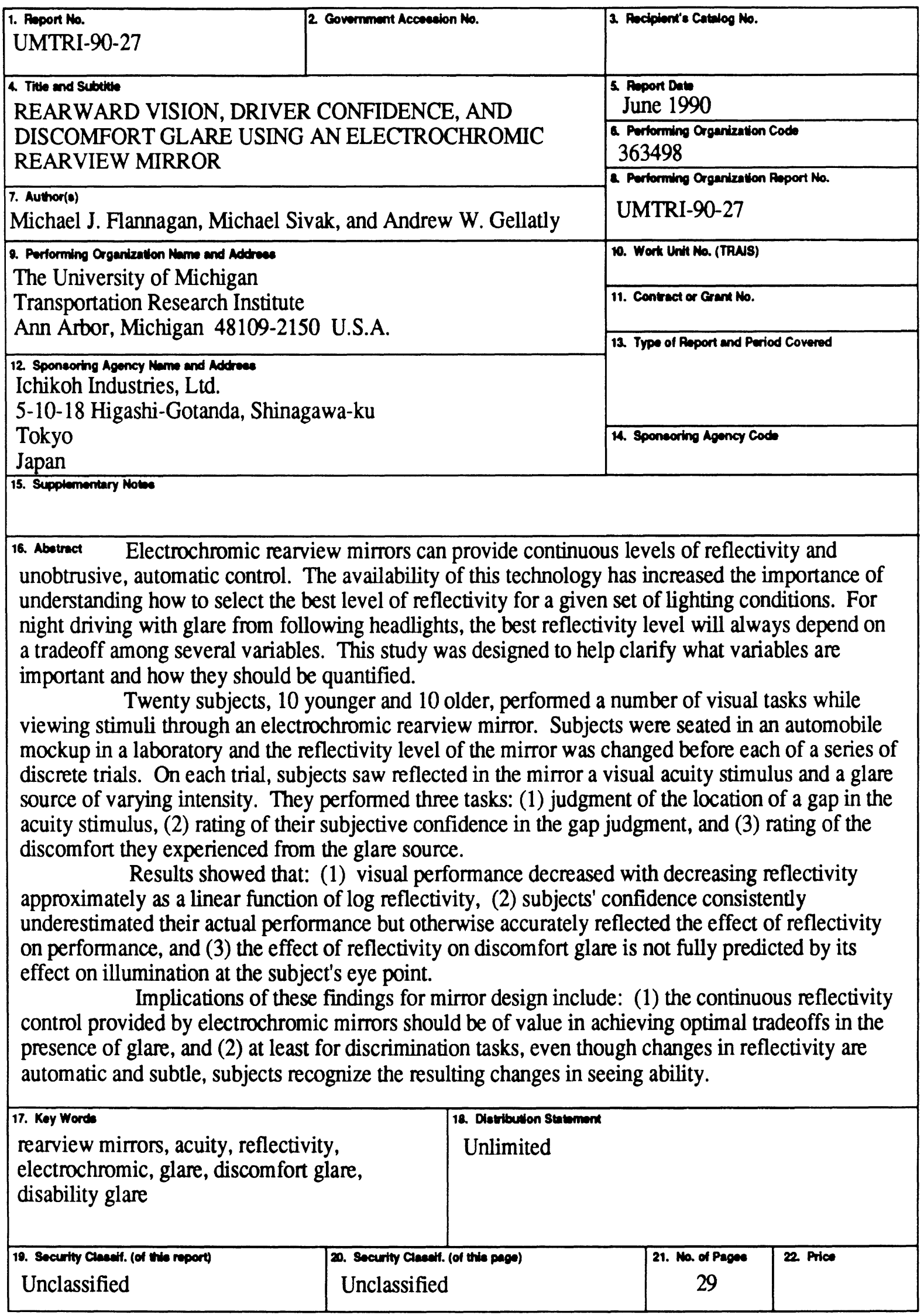




\section{ACKNOWLEDGMENT}

We wish to thank Ichikoh Industries, Ltd. for their generous support of this research. 


\section{CONTENTS}

ACKNOWLEDGMENT ......................................................

INTRODUCTION ..............................................................

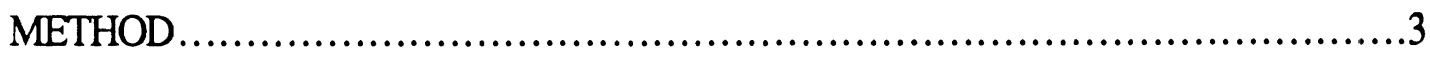

RESULTS AND DISCUSSION .................................................10

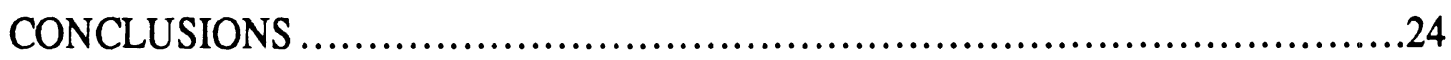

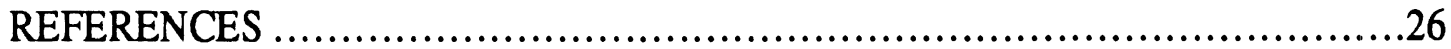




\section{INTRODUCTION}

The reflectivity levels of rearview mirrors affect at least three major aspects of vision in night driving: rearward visibility, forward visibility, and discomfort glare caused by following headlights. Just as in the cases of headlamp intensity and beam pattern, design decisions about mirror reflectivity must take into account certain tradeoffs among these variables that are foreordained by the lighting conditions of night driving. Whenever there are following headlights, higher mirror reflectivity will result in greater rearward visibility but also lower forward visibility (because of veiling glare), and greater discomfort glare. Only a modest amount of research has been undertaken to provide the data necessary to specify the optimum tradeoff among these effects (Flannagan, 1988). This may be partly because the dominant method of varying mirror reflectivity, the prism mirror, has been able to provide only very coarse control. It has been argued that the two levels of reflectivity provided by the typical prism mirror are too extreme (the high level too high and the low level too low) even to approximate the optimal tradeoff when glare is present (Olson \& Sivak, 1984, Flannagan \& Sivak, 1990). Recently, however, there has been growing interest in, and use of, mirror technologies that allow finer control of reflectivity, especially electrochromic rearview mirrors (e.g. Lynam, 1987). Electrochromic mirrors allow continuously variable reflectivity levels over a wide range, and provide the capability of simple, automatic control of reflectivity. Because these technological developments have offered the prospect of a truly satisfying optimization of mirror reflectivity, they have stimulated interest in understanding the issues involved.

The present study was motivated by the increased need to understand the effects of rearview mirror reflectivity. We began with the simple view that the problem of optimizing reflectivity fundamentally involves measuring the three variables mentioned above (forward visibility, rearward visibility, and discomfort glare) as functions of reflectivity, and determining their proper weighting. The issues addressed here should be thought of as potential complications to that simple scheme. The study had three specific goals:

The first goal was to measure rearward seeing ability as a function of mirror reflectivity employing both a wide range and reasonably fine set of steps in reflectivity. The main issue of interest here is the nature of the functions relating visual performance to reflectivity. There is some evidence (Helder, 1987; Olson, Jorgeson, \& Mortimer, 1974) that measures of visual performance such as reaction time and error rate may be strongly nonlinear functions of reflectivity. Strong nonlinearities in performance would of course have important implications for specifying tradeoffs. However, the existing evidence regarding mirror reflectivity is compatible with the possibility that performance measures are at least roughly linear with the 
logarithm of reflectivity level. If all the factors of interest are in fact log-linear with reflectivity, then reflectivity levels would still be determined by essentially linear tradeoffs.

Secondly, the study sought to measure subjects' awareness of their seeing ability as a function of mirror reflectivity. The main issue addressed by this measure is whether, in addition to the three factors mentioned above (forward visibility, rearward visibility, and discomfort), the optimization of mirror reflectivity should reflect subjective assessment of seeing ability. If people's awareness of their seeing ability closely reflects their actual ability, then such additional considerations are not necessary. But it is possible that people are not fully aware of how certain factors affect their ability to see. The changes in reflectivity of automatically controlled, continuously variable electrochromic mirrors may sometimes go unnoticed by drivers. If so, and if their ability to see is diminished at lower reflectivity as might be expected, it is possible that they will be dangerously unaware of their visual impairment. In the present study the reflectivity of a mirror was changed randomly from trial to trial without explicit notice to the subjects, roughly reflecting the level of awareness of a driver in an actual driving situation.

The third goal was to measure discomfort glare as a function of reflectivity. Along with the measurement of rearward visibility, this allows us to explore the tradeoff between two of the three major variables that we believed to be important in determining optimal reflectivity. An additional issue addressed by this measure is the possibility that visibility influences discomfort glare ratings, meaning that the three factors involved in the tradeoff as outlined above are not easily separated. There is evidence that task difficulty influences discomfort glare ratings (Sivak, Flannagan, Ensing, \& Simmons, 1989), and rearview mirror reflectivity presents a particularly interesting case in which to investigate this effect because changing reflectivity inherently links reducing illumination at the eye (which should reduce discomfort) with reduced ability to perform a rearward visual task (which should increase discomfort). 


\section{METHOD}

\section{Tasks}

Three tasks were performed on each trial. First, the subject performed a gap-location task, deciding whether a gap had appeared in the top or the bottom of a briefly projected outline square. Second, the subject rated his or her confidence in the judgment of gap location for that trial. Third, the subject gave a numerical rating of the discomfort experienced from a light source which accompanied the presentation of the gap-location stimulus.

\section{Equipment}

Schematic diagrams of the experimental setup and the subject's view are shown in Figures 1, 2, and 3. The subject was seated in a mockup of a 1985 Chrysler Laser equipped with an electrochromic rearview mirror. The mirror was connected to a voltage source that allowed any one of five preset voltages to be applied to the mirror, thereby setting its reflectivity at one of five levels. The reflectivity levels were chosen to be spaced approximately equally on a logarithmic scale: $0.80,0.39,0.21,0.11$, and 0.054 . In front of the subject, at a distance of $7.3 \mathrm{~m}$, was a blank wall diffusely illuminated by a small incandescent lamp which was just in front of the mockup and below the subject's direct line of sight. Except when stimuli were briefly presented during a trial, there was no other significant source of light in the room. The luminance of the front wall was $3.40 \times 10^{-2} \mathrm{~cd} / \mathrm{m}^{2}$.

Behind the subject, and visible through the rearview mirror, was a rear-projection screen. The distance from the subject's eyes, through the rearview mirror, to the screen was $6.1 \mathrm{~m}$. Black tape $(1.9 \mathrm{~cm}$ wide) delineated a $28 \times 28 \mathrm{~cm}$ area at the edge of the screen that was toward the subject's right as the subject sat facing away from the screen (i.e., the right edge of the screen as viewed by the subject through the rearview mirror). Two small red LEDs were located on the border of that area, one at the middle of the top edge and one at the middle of the bottom edge. The rear-projection screen was illuminated by light scattered from the front wall and had a luminance of $1.34 \times 10^{-3} \mathrm{~cd} / \mathrm{m}^{2}$. The LEDs, as viewed by the subject, had luminous intensities of about $1.50 \times 10^{-3} \mathrm{~cd}$.

A random-access slide projector located behind the screen was used to project a gaplocation stimulus (described below) into the center of the taped square. A second randomaccess slide projector, immediately adjacent to the left edge of the screen, provided the glare source. The center-to-center distance between the glare source and the projected gap-location stimulus was $26.7 \mathrm{~cm}$ (a visual angle of 2.5 degrees as viewed by the subject). Neutral density filters mounted in slide holders allowed the intensity of the glare source in the direction of the rearview mirror to be $139,13.6$, or $1.20 \mathrm{~cd}$. Both projectors were equipped with automatic, 
remote-controlled shutters which were electronically coupled to provide synchronous 2 -second presentations of the glare light and the gap-location stimulus. The openings of the shutters were each $2.54 \mathrm{~cm}$ in diameter. The glare source thus subtended 14 minutes of arc at the subject's eye point.

A large panel with a 9-point rating scale for discomfort glare, printed in black letters on a white background, was posted in front of the subject at a distance of $3.4 \mathrm{~m}$. The letters were $1.9 \mathrm{~cm}$ high and subtended approximately 19 minutes of arc. This chart was offset so that it appeared to the left of the subject's forward field of view, which was otherwise just the evenly illuminated blank wall of the laboratory. 


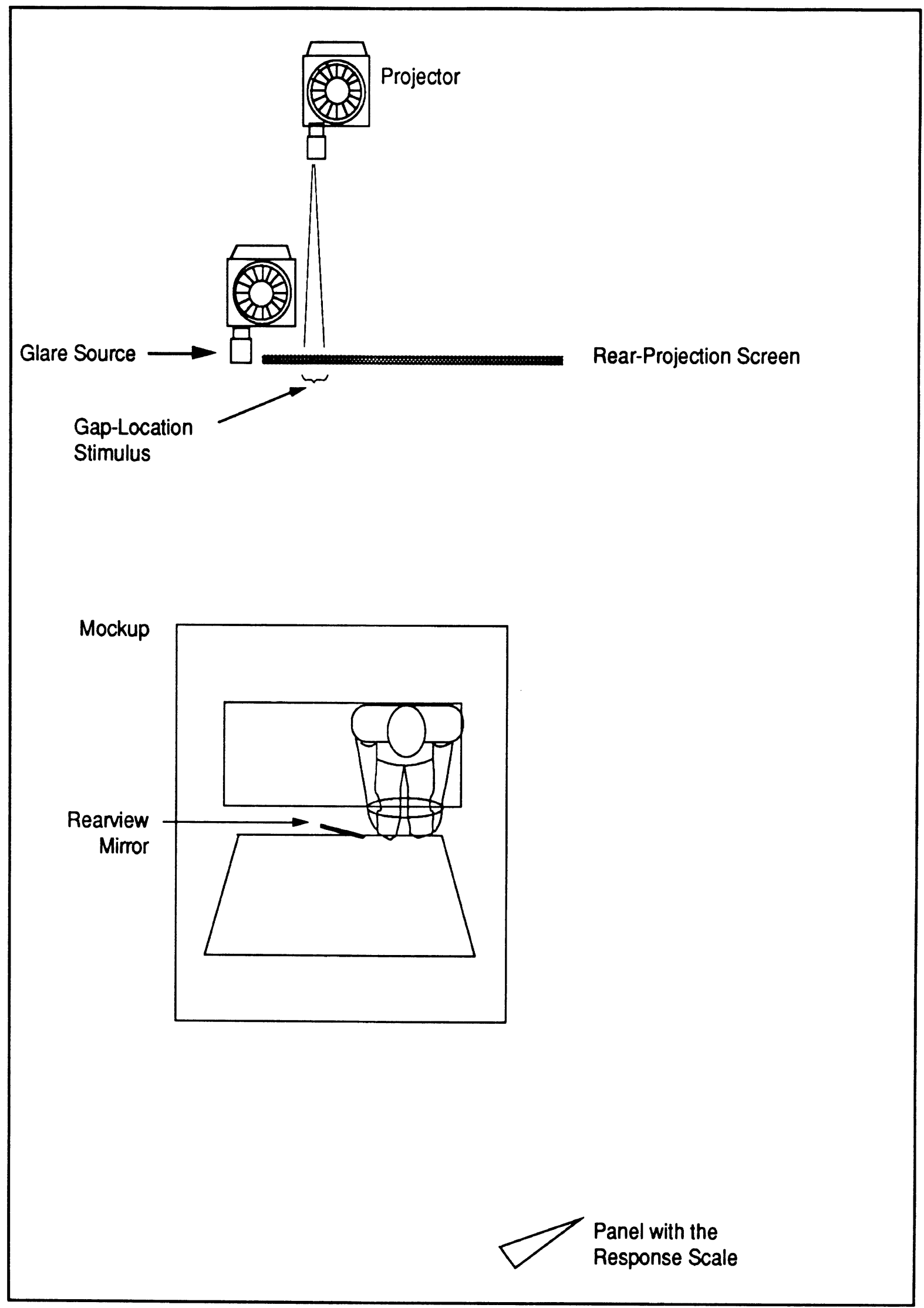

Figure 1. A schematic diagram of the experimental setup (distances are not to scale). 


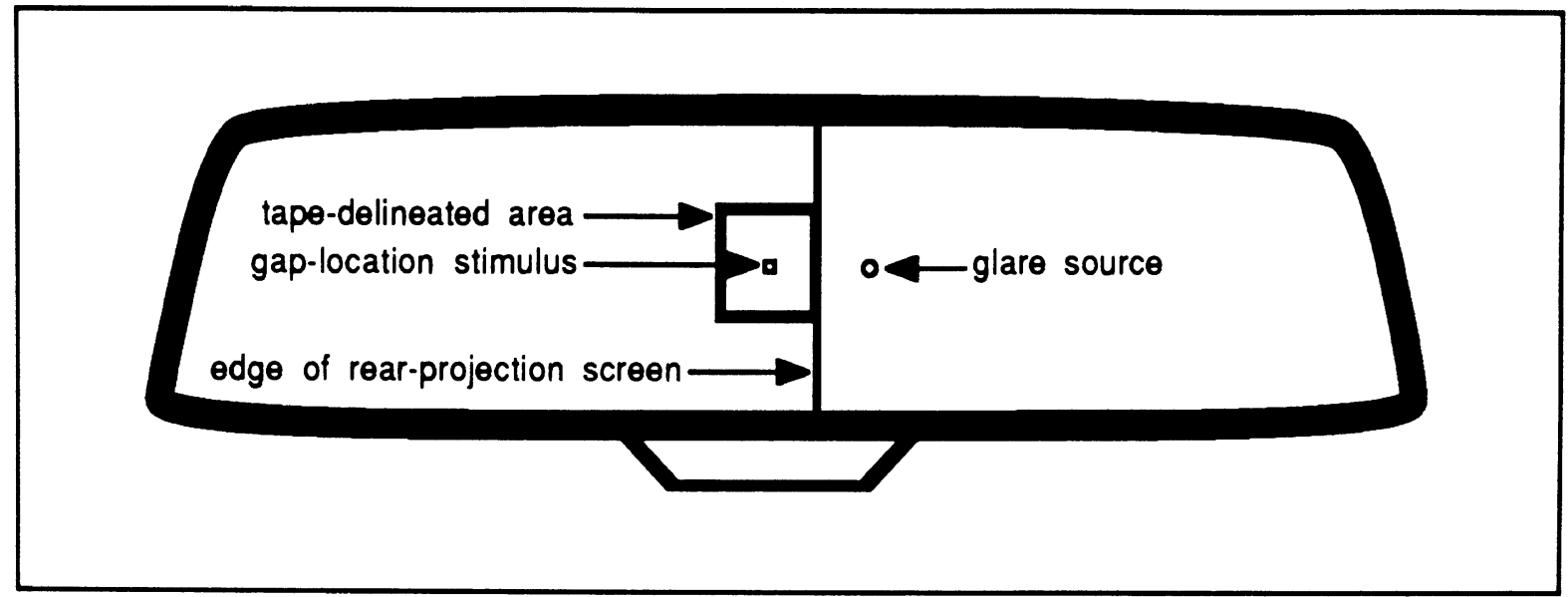

Figure 2. The subject's view of the rearview mirror, showing the spatial relationships of the stimuli as seen reflected in the mirror.

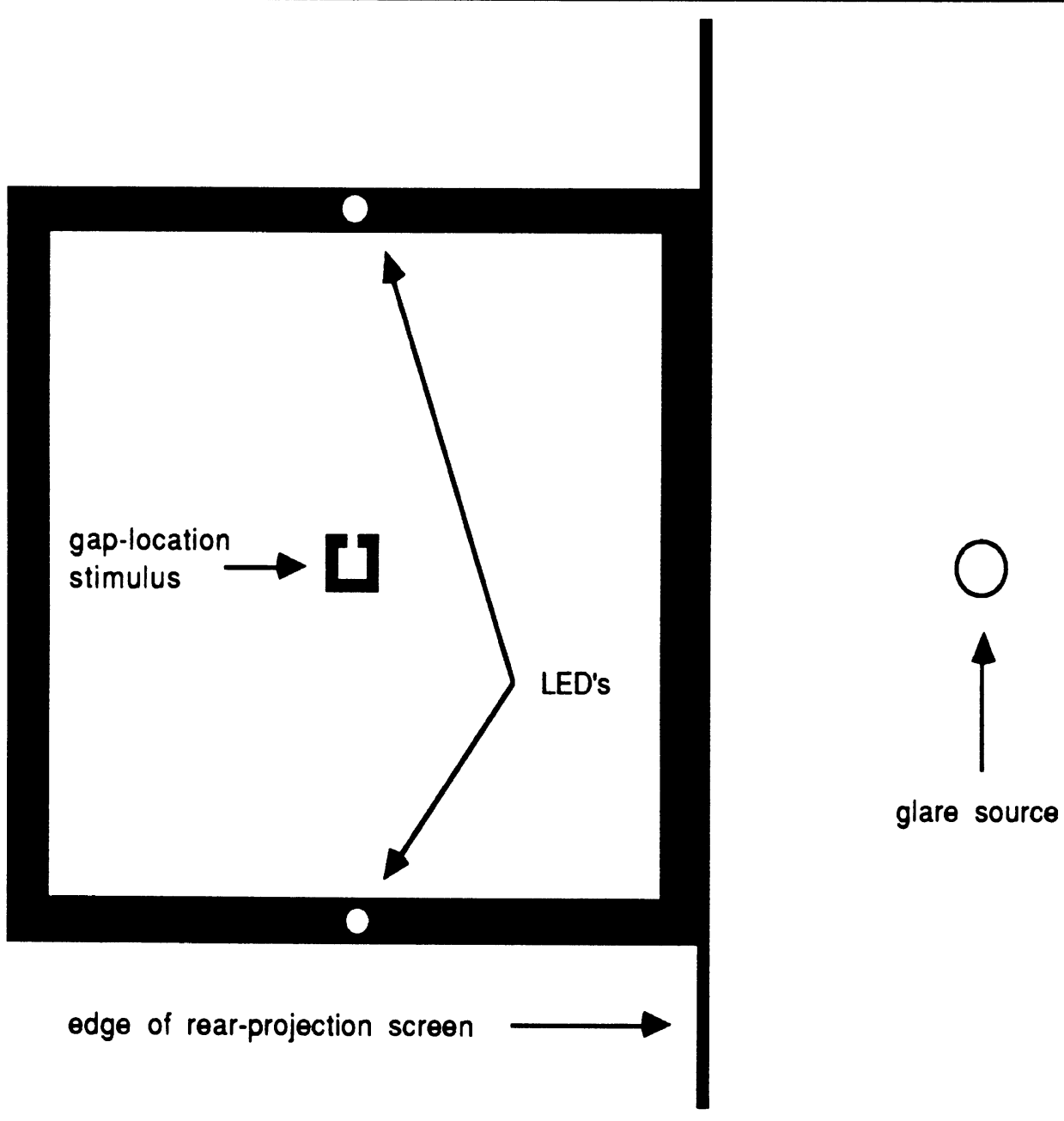

Figure 3. Detail of subject's view through the rearview mirror. 


\section{Gap-location stimuli}

The stimuli for the gap-location task were outlines of squares subtending 10.2 minutes of arc in height and width, with a stroke width of 2.3 minutes. The gap appeared in the middle of either the top or bottom edge and was 1.7 minutes of arc wide. The outline squares appeared as white figures on a dark background. The luminance of the strokes of the squares was $2.70 \mathrm{~cd} / \mathrm{m}^{2}$. The background luminance was $1.34 \times 10^{-3} \mathrm{~cd} / \mathrm{m}^{2}$.

\section{Glare levels}

Fifteen glare levels were produced by the factorial combination of the five rearview mirror reflectivities and the three glare source intensities described above. The lux values at the subject's eye point for those fifteen levels are given in Table 1.

\section{Table 1}

Lux values at the subject's eye point for the fifteen levels of glare.

\begin{tabular}{l|l|l|l}
\hline \multirow{2}{*}{ Reflectivity } & \multicolumn{3}{|c}{ Glare source intensity (cd) } \\
\cline { 2 - 4 } & 139 & 13.6 & 1.20 \\
\hline 0.80 & 3.00 & 0.293 & 0.0257 \\
0.39 & 1.49 & 0.145 & 0.0127 \\
0.21 & 0.793 & 0.0774 & 0.00679 \\
0.11 & 0.408 & 0.0398 & 0.00349 \\
0.054 & 0.204 & 0.0199 & 0.00175 \\
\hline \hline
\end{tabular}

\section{Daily photometry checks}

To insure that photometric levels remained stable over the period in which data were collected, ambient illumination and glare levels were checked at the beginning of each day's session. The coefficient of variation (standard deviation divided by the mean) for those measures averaged 3.0 percent. 


\section{Response scale}

Subjects were asked to use a 9-point scale for their assessment of discomfort glare. This scale has been used extensively to evaluate glare in night driving situations (e.g., Bhise, Swigart, \& Farber, 1975; de Boer, 1967). It has qualifiers only for the odd points as follows: 1 (unbearable), 2, 3 (disturbing), 4, 5 (just acceptable), 6, 7 (satisfactory), 8, 9 (just noticeable).

\section{Confidence ratings}

Subjects were asked to rate their confidence in each gap-location judgment by picking a number from 50 to 100 . They were told that the number should represent the number of times that they would expect to be correct if they performed 100 hypothetical trials, all of difficulty equal to the trial that they were rating. Thus a subject should choose 100 if he or she was certain that a gap judgment was correct, 50 if the judgment was a complete guess, or any number in between to represent intermediate states of confidence.

\section{Subjects}

Twenty paid subjects participated in this study. There were five subjects in each of the following groups: younger males, younger females, older males, and older females. The ages of the younger subjects ranged from 20 to 27 with a mean of 22.6 years, and the ages of the older subjects ranged from 64 to 77 with a mean of 69.7 years. Subjects were selected to have visual acuity of 20/30 or better as measured by a test using Landolt rings. The range of visual acuity for the younger group was 20/13 to 20/30 and the range for the older group was 20/17 to 20/30. In order to reduce ceiling or floor effects in the difficulty of the gap-location task, any subject whose overall percent correct on that task was greater than $95 \%$ or less than $55 \%$ (with $50 \%$ being chance performance) was replaced. Four younger subjects who were above 95\% and five older subjects who were below 55\% were replaced in arriving at the twenty subjects for whom results are reported here.

\section{Design}

There were 30 trial types ( 3 glare intensities $x 5$ mirror reflectivities $x 2$ gap positions). A block of trials consisted of a random arrangement of these 30 trial types. Each subject was given a set of 6 practice trials followed by 3 experimental blocks of trials.

\section{Procedure}

Subjects were tested individually. Subjects were told that on each trial a small white outline of a square would appear for two seconds in the center of the taped projection area of 
the rear-projection screen. The square would contain a small gap in the center of either the top or the bottom edge. In addition, there would be a light to the right of the stimulus as seen through the mirror. The square and the light appeared simultaneously for two seconds. Subjects were instructed to look at the laboratory wall in front of them, rather than at the mirror, in between trials. Just before each trial the experimenter said "ready," at which point the subject was to look into the mirror and at the center of the taped area in order to be ready for the gap-location stimulus when it appeared. The two red LEDs mounted on the tape border provided additional visual landmarks to help the subject in locating the position where the stimulus would appear. The interval between trials was fixed at 40 seconds, an interval long enough to insure that the mirror had stabilized at the reflectivity selected for the upcoming trial.

On each trial subjects responded verbally by first saying "up" or "down" to indicate their judgment about the location of the gap, then a number ( 50 to 100) to indicate their degree of confidence in the location judgment, and finally a number (1 to 9 ) to indicate the subjective discomfort from the glare source. At the start of data collection subjects had been adapted for about 15 minutes to the prevailing ambient illumination; at its completion they had been adapted for about 75 minutes. 


\section{RESULTS AND DISCUSSION}

The results of this study will be presented in two sets: (1) the effects of the independent variables (primarily glare intensity and mirror reflectivity) on each of the three dependent variables (percent correct on gap location, confidence regarding gap location, and rating of discomfort glare) individually, and (2) relationships among the three dependent variables.

\section{Effects on individual dependent variables}

Data for percent correct, confidence, and discomfort rating were submitted to three parallel analyses of variance using the following factors: glare intensity, mirror reflectivity, direction of gap (up or down), experimental block (the first, second, or third set of 30 trials in the procedure for one subject), sex, and age group (younger or older).

Percent correct. In the analysis of percent correct, three main effects were significant: glare intensity, $F(2,32)=104.28, p<.0001$, reflectivity, $F(4,64)=9.09$, $p<.0001$, and age, $F(1,16)=27.73, p<.001$. Only two interactions were significant: the two-way interaction of age with glare, $F(2,32)=6.50, p<.01$, and the three-way interaction of age, glare, and reflectivity, $F(8,128)=3.59, p<.001$.

Both of the main effects of reflectivity and glare, and the lack of an interaction between them, can be seen in Figure 4. Percent correct goes down as glare intensity increases and as mirror reflectivity decreases. The three curves are parallel, indicating that the effect of reflectivity is the same for each level of glare intensity. At each level of glare source intensity, the effect of mirror reflectivity on percent correct is roughly linear with the logarithm of reflectivity. (Note that the abscissa in Figure 4, and in most subsequent figures, is logarithmic.)

Interpretations of the main effect of age on percent correct should take into account two aspects of how these subjects were selected. First, older and younger subjects were closely matched on standard visual acuity (measured with high ambient illumination and no glare), and second, several subjects were excluded from analysis because their performance over all combinations of glare intensity and reflectivity was too uniformly low or too uniformly high to allow differentiation of those treatments. All four of the subjects excluded because they were below the minimum overall-performance criterion were in the older group, and all five of the subjects excluded because they were above the maximum overall-performance criterion were in the younger group. However, in spite of acuity matching and trimming of extreme performance, the mean percent correct for the younger group (87.0) was substantially higher than that for the older group (72.2). This difference might be considered surprising because 
the gap-location task is very similar to the standard acuity test on which the age groups were matched. However, the experimental task was done at lower luminance levels and in the presence of glare, either or both of which may have been responsible for worse performance by the older group. Indeed, the significant interaction of age and glare, shown in Figure 5, indicates that the older group began to experience difficulty at a lower level of glare than the younger group. At the intermediate glare level the younger group is no worse than at the lowest level, but the older group shows a substantial deficit.

The three-way interaction of age, glare, and reflectivity is shown in Figure 6. Although the data are quite variable at this level of detail, the character of the interaction seems clear. The older group appears to show a floor effect in the highest glare intensity condition; for all reflectivities their performance is near chance. In contrast, the younger group shows a ceiling effect in the two lower glare intensity conditions; their performance is nearly perfect for the three highest levels of reflectivity. In spite of those ceiling and floor effects, however, the younger and older groups both show a marked effect of reflectivity on gap-location performance, and in both cases the effect appears to be linear except for the influence of the scale limits. 


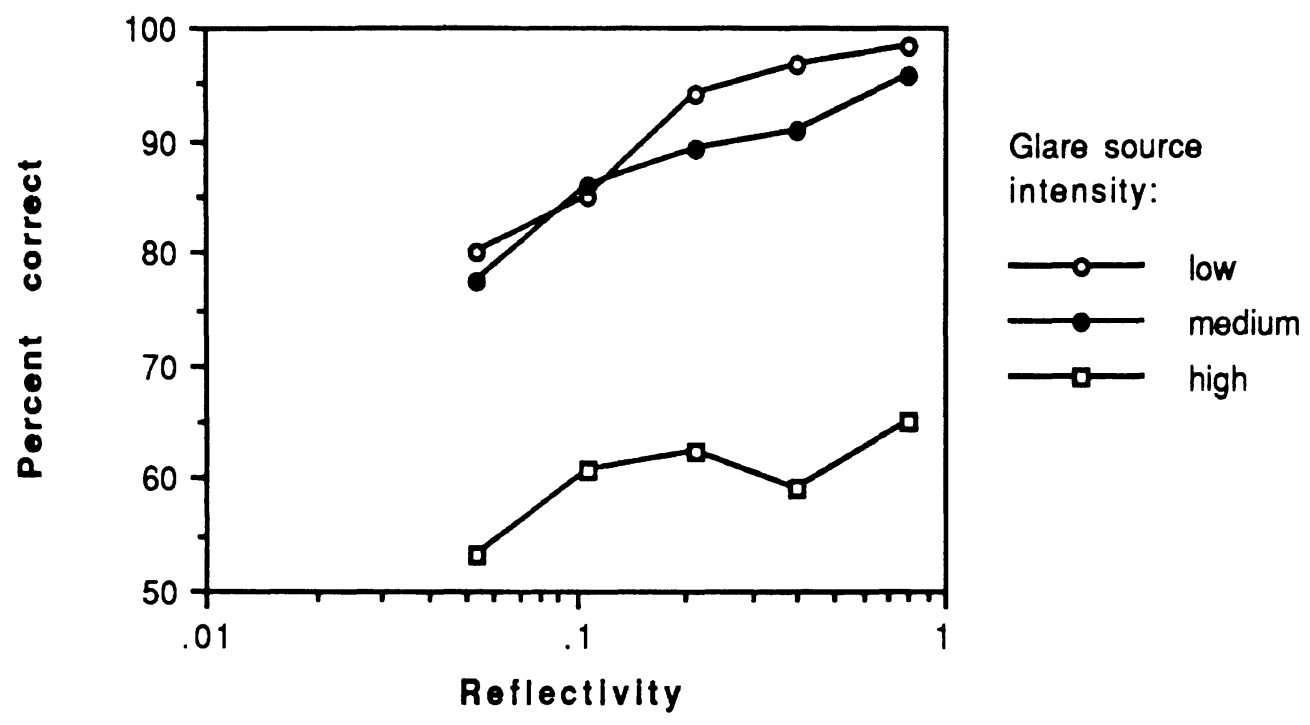

Figure 4. Percent correct on the gap-location task for each combination of reflectivity and glare source intensity.

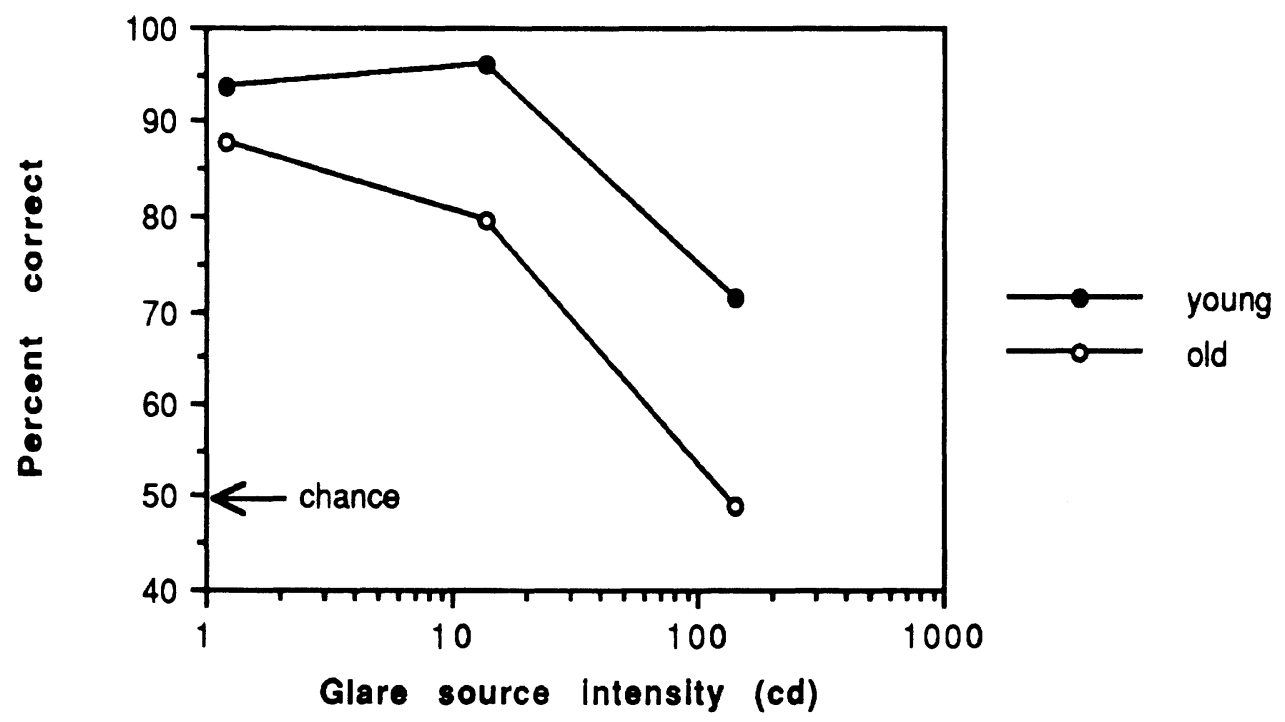

Figure 5. The interaction of age and glare source intensity for percent correct on the gap-location task. 

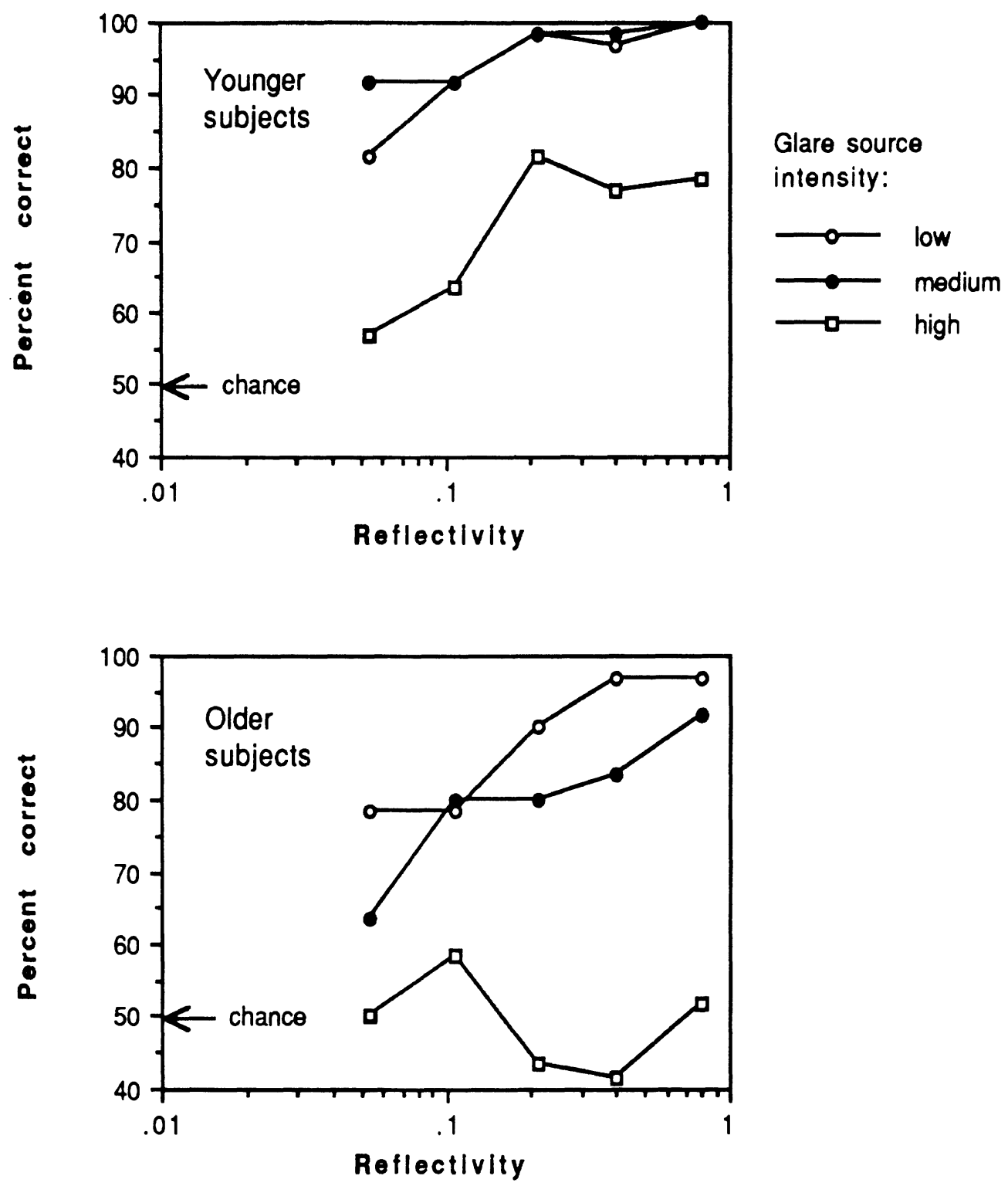

Figure 6. The three-way interaction of age, glare source intensity, and reflectivity for percent correct in the gap-location task. 
Confidence. In the analysis of confidence ratings, two main effects were significant: glare intensity, $F(2,32)=101.66, p<.0001$, and reflectivity, $F(4,64)=123.86, p<.0001$. Only one interaction was significant: the two-way interaction of glare with reflectivity, $F(2,32)$ $=6.50, p<.0001$. The main effects of both glare intensity and reflectivity and their interaction can all be seen in Figure 7. Confidence increases with decreasing glare and with increasing reflectivity. The interaction of glare and reflectivity is such that reflectivity has a much greater effect on confidence for low and medium glare intensity than for high glare intensity. Although the slopes of the lines for the low and medium glare intensity conditions are strongly positive, the slope of the high glare intensity line is substantially lower and nearly flat. As with the interactions that were significant in the analysis of percent correct, the graph of this interaction suggests that it can be thought of as a floor effect. A confidence rating of 50 was the lowest response permitted, and was supposed to indicate that a subject believed a gap judgment was a complete guess. It appears subjects believed that nearly all gap judgments with high intensity glare were guesses. Unlike the low and medium glare intensity conditions, with high glare intensity lower reflectivity could not decrease their confidence because even at high reflectivity they thought they were guessing.

Discomfort. In the analysis of discomfort ratings, two main effects were significant: glare intensity, $F(2,32)=378.86, p<.0001$, and reflectivity, $F(4,64)=19.64, p<.0001$. Only one interaction was significant: the two-way interaction of glare with reflectivity, $F(2,32)$ $=6.50, p<.0001$. Interestingly, these three effects are the same three that were significant in the analysis of confidence ratings. All three effects on discomfort glare ratings can be seen in Figure 8. Discomfort ratings decrease numerically (indicating more discomfort) as glare source intensity increases and as mirror reflectivity increases. As indicated by the significant interaction between glare and reflectivity, the three lines for the glare conditions are not parallel. The slope of the line for the high glare intensity condition is more negative than the slopes of the other two lines, which are roughly parallel to each other. Unlike all of the interactions discussed above, this effect cannot be caused by experimental conditions reaching an upper or lower limit of the dependent variable (i.e., ceiling or floor effects). In fact there may be a floor effect working against the interaction. Discomfort ratings for the higher reflectivity levels at high glare intensity are close to the lower limit of the rating scale (a value of 1 ). Thus those values might have been even lower (increasing the disparity in slope for the high glare intensity condition versus the other two) if not for the influence of the scale boundary. A possible explanation for this pattern of results, involving a relationship between discomfort ratings and confidence ratings, will be suggested below in the discussion of relationships among dependent variables. 


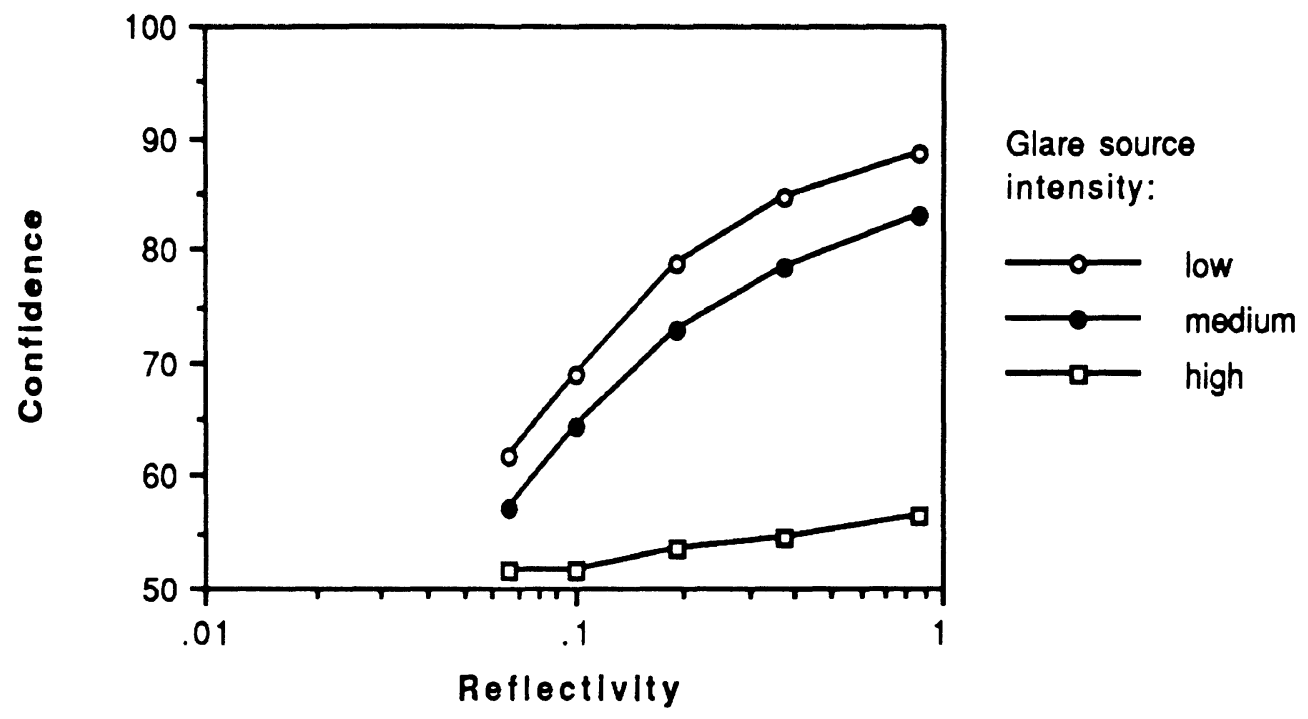

Figure 7. Ratings of confidence on the gap-location task for each combination of reflectivity and glare source intensity.

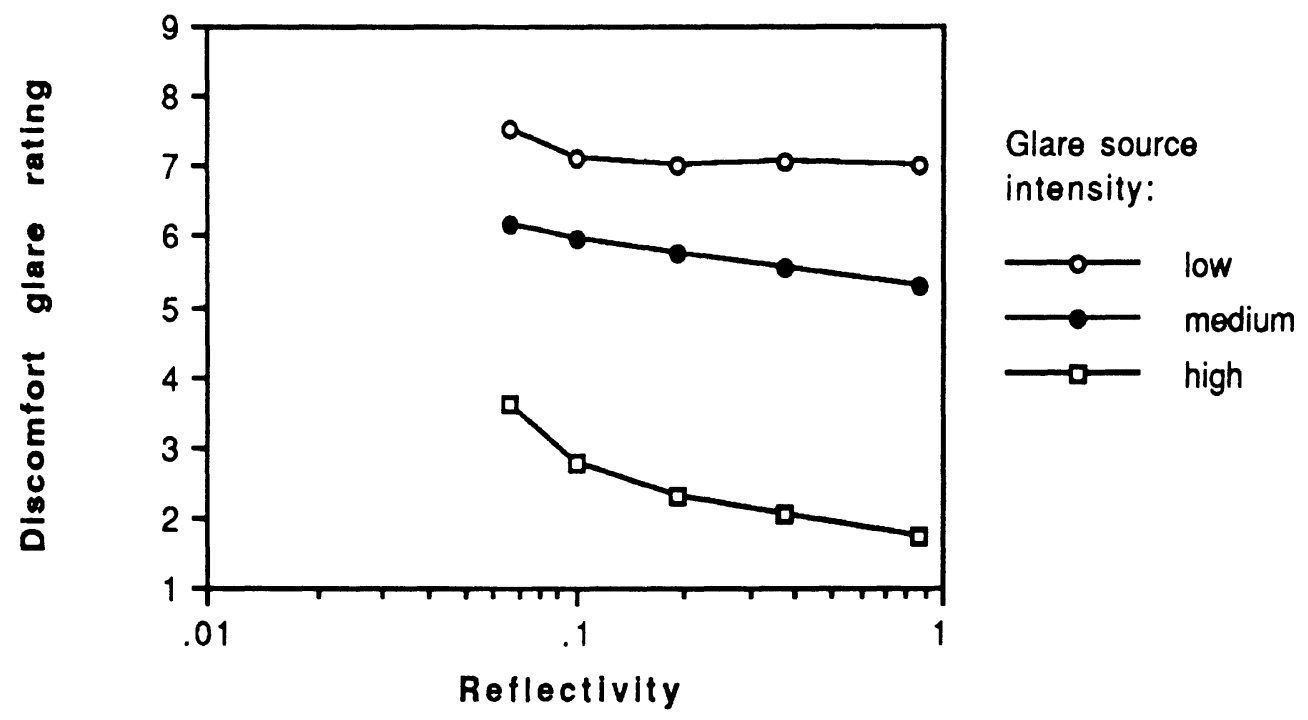

Figure 8. Discomfort glare ratings for each combination of reflectivity and glare source intensity. 
Both mirror reflectivity and glare source intensity affect the illumination at the subject's eye point, as indicated in Table 1. In the case of discomfort glare ratings it is worth considering the possibility that the effects of reflectivity and glare source intensity can both be understood as effects of illumination. Indeed, one widely used model of discomfort glare suggests that should be the case (Schmidt-Clausen \& Bindels, 1974). According to that model, discomfort glare ratings are predicted by

$$
\mathrm{W}=5.0-2 \log \frac{\mathrm{E}_{\mathrm{B}}}{\mathrm{C}_{\mathrm{poo}}\left[1+\sqrt{\frac{\mathrm{LU}_{\mathrm{U}}}{\mathrm{C}_{\mathrm{pL}}}}\right] \theta^{0.46}}
$$

where $\mathrm{W}$ is a discomfort glare rating on the de Boer scale, $\mathrm{E}_{\mathrm{B}}$ is the illumination at the observer's eye point in lux, $\mathrm{C}_{\mathrm{poo}}$ is a constant equal to $3.0 \times 10^{-3} \operatorname{lux} \min ^{-0.46}, \mathrm{~L}_{\mathrm{U}}$ is the luminance to which the observer is adapted in candelas per square meter, $\mathrm{C}_{\mathrm{pL}}$ is a constant equal to $4.0 \times 10^{-2} \mathrm{~cd} \mathrm{~m}^{-2}$, and $\theta$ is the visual angle in minutes between the glare source and the observer's visual fixation point. In the procedure used here adaptation luminance and visual angle of the glare source were held constant; thus discomfort ratings should be predictable directly from illuminance values.

To explore that possibility, the values of the dependent variables for each combination of glare intensity and reflectivity are plotted in Figures 9, 10, and 11 as functions of the illuminance at the subject's eye. These are the same data as in Figures 4, 7, and 8, simply replotted to clarify their relationship to illuminance. To facilitate comparison with the earlier figures the data points for each glare source intensity condition are joined. Not surprisingly, in the cases of percent correct and confidence rating lux value is not a very good predictor. Both measures increase with illumination for sets of conditions over which reflectivity varies and for which glare source intensity is constant. In contrast, both measures decrease with illumination for sets of conditions over which glare source intensity varies and for which reflectivity is constant. The situation for discomfort glare ratings, in which a simple relationship with illumination can be expected, is more interesting. The relationship predicted by the Schmidt-Clausen and Bindels model is shown in Figure 11. $\mathrm{L}_{U}$ has been set to the value of the luminance of the front wall, $0.034 \mathrm{~cd} / \mathrm{m}^{2}$, and $\theta$ has been set to the visual angle between the gap-location stimulus and the glare source, 150 minutes. Overall there is good agreement between the model and the data. For any set of conditions in which mirror reflectivity is constant (such as the three points that are leftmost in each of the three groups joined by lines), discomfort ratings are roughly linear with the logarithm of lux value and the slope is roughly as 
predicted. For the three sets of conditions distinguished by glare source intensity, however, only the set with the highest intensity is in rough agreement with the model. When glare source intensity is medium or low the slopes of the data are distinctly less negative than predicted. A revised model for these data, incorporating rated confidence as a predictor, is presented below.

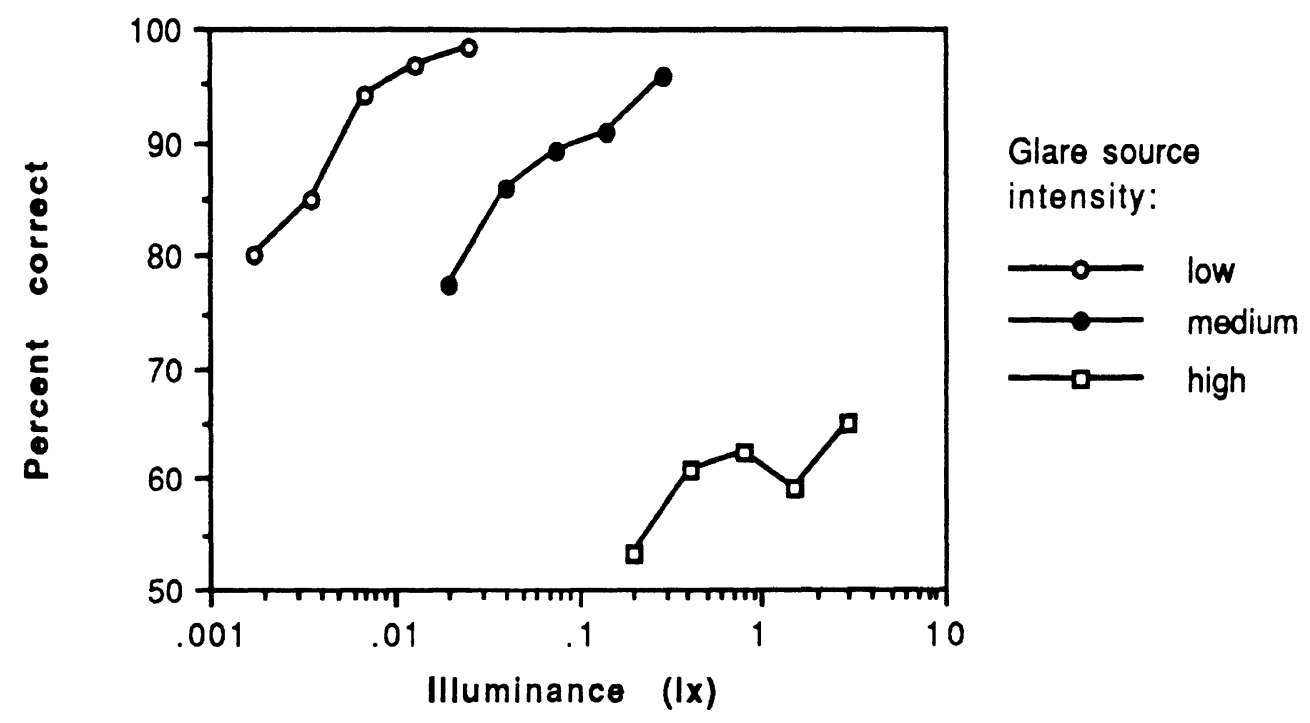

Figure 9. Percent correct on the gap-location task for each combination of reflectivity and glare source intensity, plotted by illuminance at the subject's eye point.

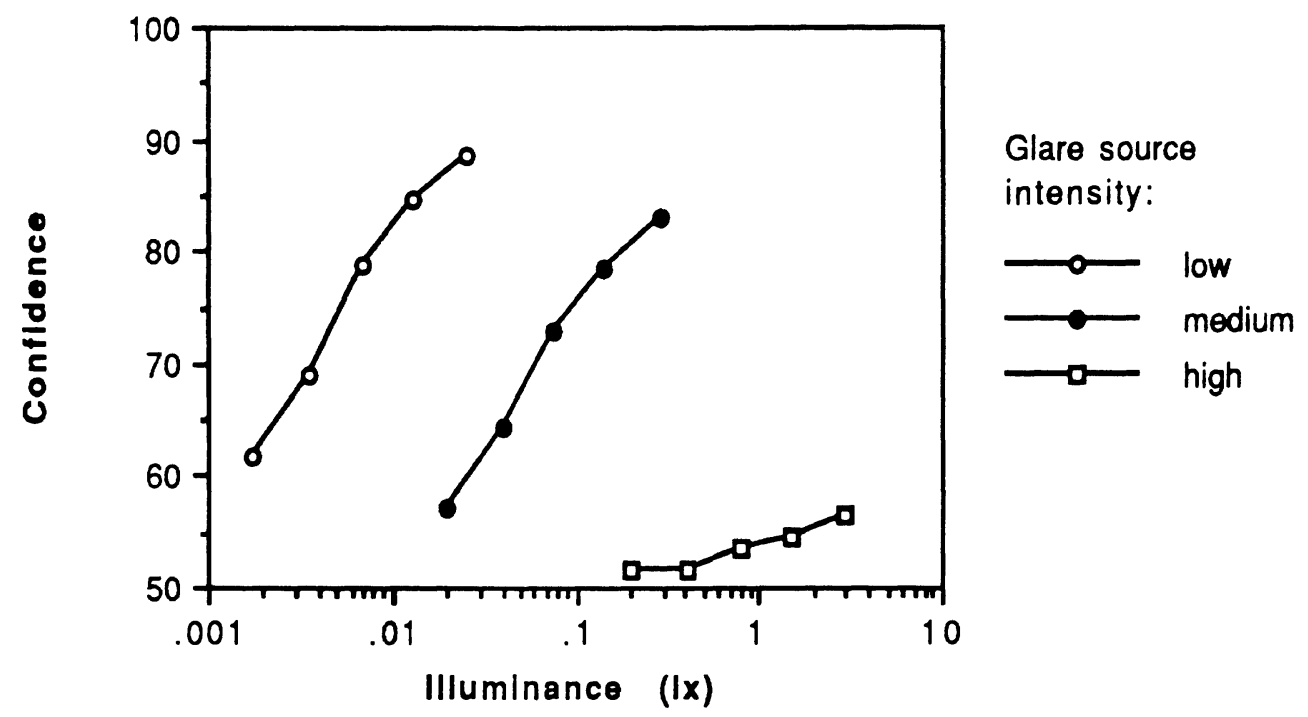

Figure 10. Ratings of confidence on the gap-location task for each combination of reflectivity and glare source intensity, plotted against illuminance at the subject's eye point. 


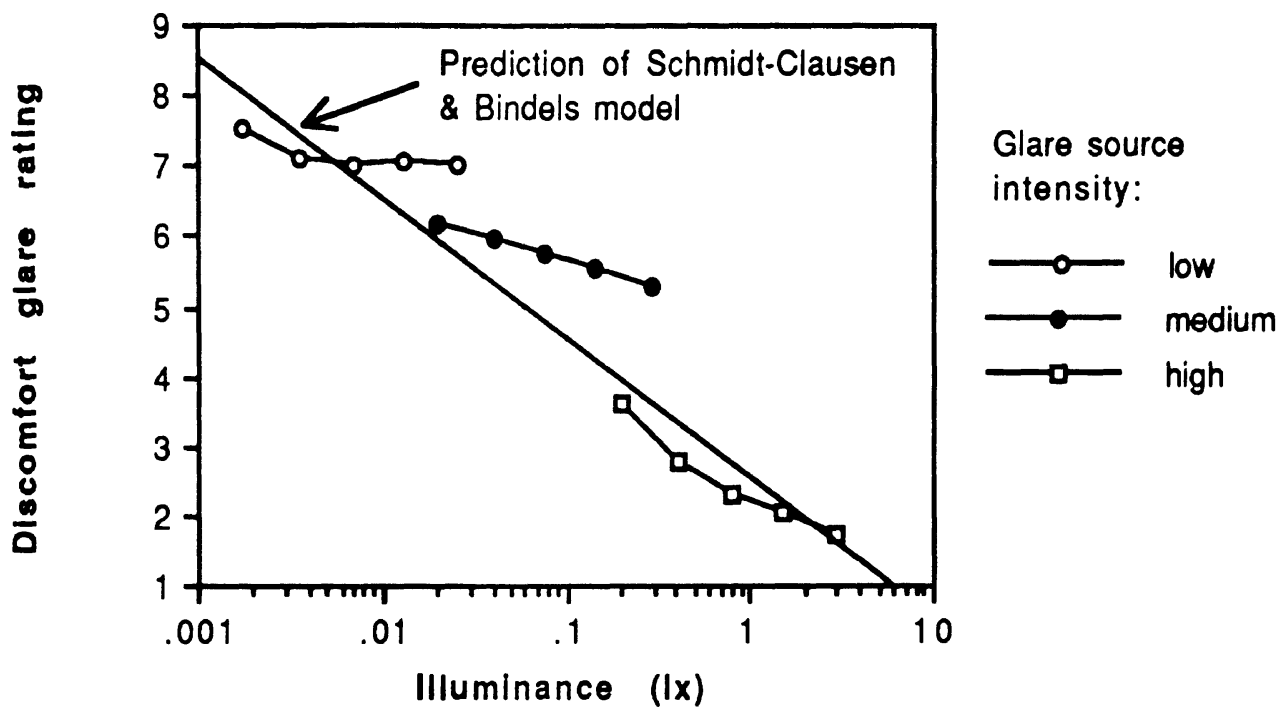

Figure 11. Discomfort glare ratings for each combination of reflectivity and glare source intensity, plotted against illuminance at the subject's eye point. 


\section{Relationships among dependent variables}

Confidence and percent correct. The relationship between subjects' confidence ratings and their actual performance is of interest primarily because of the information it provides about subjects' awareness of the visual impairment that they experience with lower reflectivities. The most interesting conditions over which to observe the relationship between confidence and performance are therefore the five levels of mirror reflectivity. The filled circles in Figure 12 show that relationship. The data plotted are the mean percent correct and confidence rating for each reflectivity level, collapsed over all other variables in the design. If subjects' confidence ratings perfectly reflected their actual performance, the data points would lie on the diagonal line with unit slope connecting the lower left and upper right corners of the diagram. The actual data indicate that subjects consistently underestimate their percent correct, but that they do recognize the effect of mirror reflectivity on their ability to perform the visual task. The slope of the best fitting line is slightly greater than one, suggesting that subjects may actually overestimate the effect of reflectivity.

Although they are of secondary interest, it is worth noting the relationships between confidence and performance over two other factors that affected performance: glare source intensity and individual subject. The relationship over glare source intensities is shown by the open squares in Figure 12. The same data have been used to determine these three points as were used to determine the five points for mirror reflectivities, but this time they have been collapsed over all variables except glare source intensity. Once again subjects' ratings consistently underestimate their performance, but otherwise reflect the effect of glare reasonably well. This time the slope is less than one, which could be interpreted as indicating that the subjects underestimated the importance of glare source intensity in determining performance. However, it is possible to argue that the slope is distorted because of subjects' overall tendency to underestimate their performance combined with the fact that the point with the lowest mean confidence rating (which corresponds to the highest glare source intensity) is against the end of the scale and so cannot be lower.

The relationship between confidence ratings and performance across individual subjects in shown in Figure 13. Although some subjects underestimate their performance more than others, all subjects show at least some underestimation. Across all subjects there is a significant correlation between confidence and performance, $r=.65, p<.01$. Across individuals within the age groups there is a significant relationship in the older group, $r=.82$, $p<.01$, but not in the younger group, $r=.23, p>.50$. 


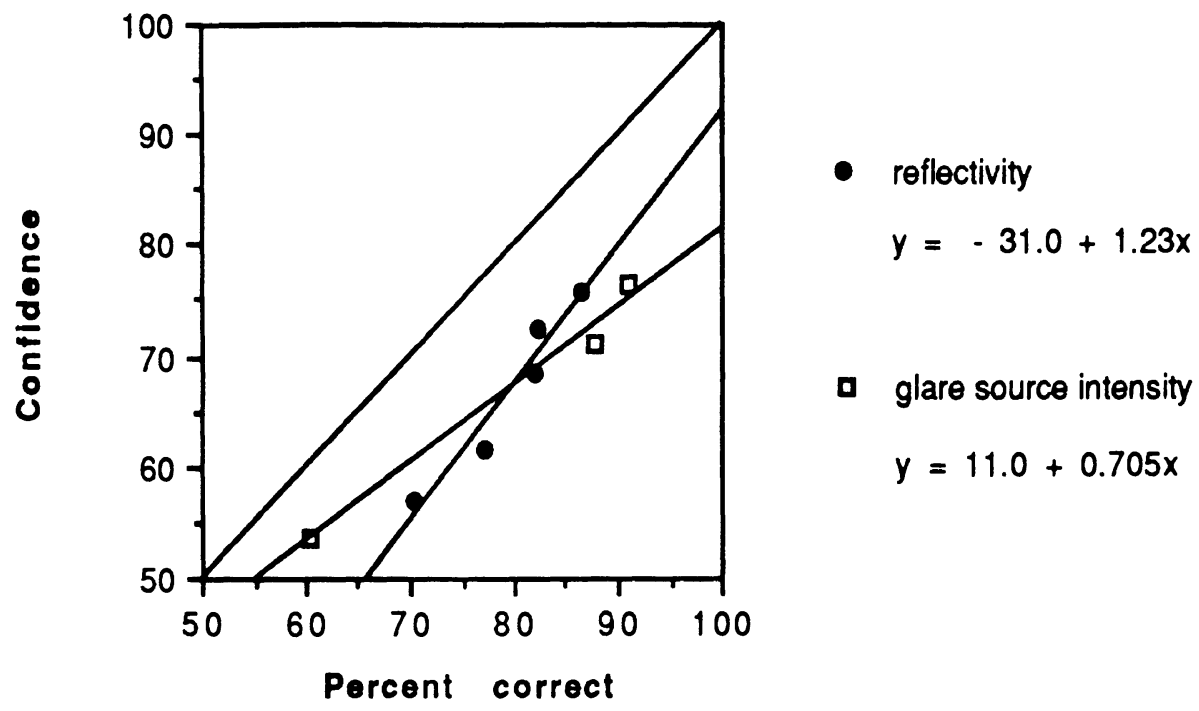

Figure 12. Confidence ratings and percent correct for the five levels of mirror reflectivity and the three levels of glare source intensity. Equations are for the regression lines shown.

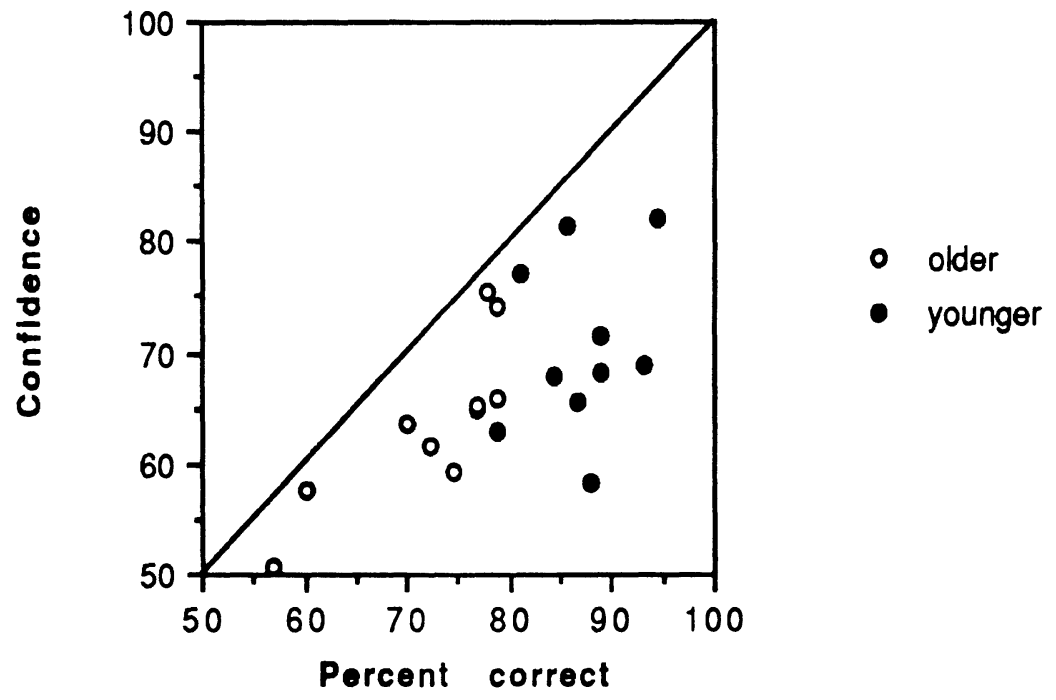

Figure 13. Confidence ratings and percent correct for the twenty individual subjects. 
Percent correct and discomfort glare ratings. In the discussions of individual dependent variables it became clear that within each of the three conditions distinguished by glare source intensity percent correct and discomfort glare ratings were both approximately linear with $\log$ reflectivity. As $\log$ reflectivity increased, percent correct increased and discomfort glare ratings decreased (indicating more discomfort). This implies that the tradeoff between these two variables, over changes in reflectivity, will be described by a linear function. When that tradeoff is plotted, as in Figure 14, that does turn out to be the case. Figure 14 is a plot of percent correct against discomfort rating for each combination of glare source intensity and reflectivity. Reflectivity levels are not identified in the figure, but as might be expected, the points within each glare source intensity group decrease in reflectivity from left to right. For a fixed level of glare source intensity, as reflectivity decreases the glare becomes less bothersome (ratings are numerically higher), but the subject's ability to perform the gap-location task is reduced. For each level of glare source intensity the tradeoff is linear. Regression lines for each of those groups are also shown. Interestingly, the slope of the tradeoff function is markedly less steep for the highest glare source intensity condition than for the other two. This means that, at high glare source intensity levels, achieving a particular reduction in discomfort is less costly in terms of lost ability to see than it is at lower glare levels.

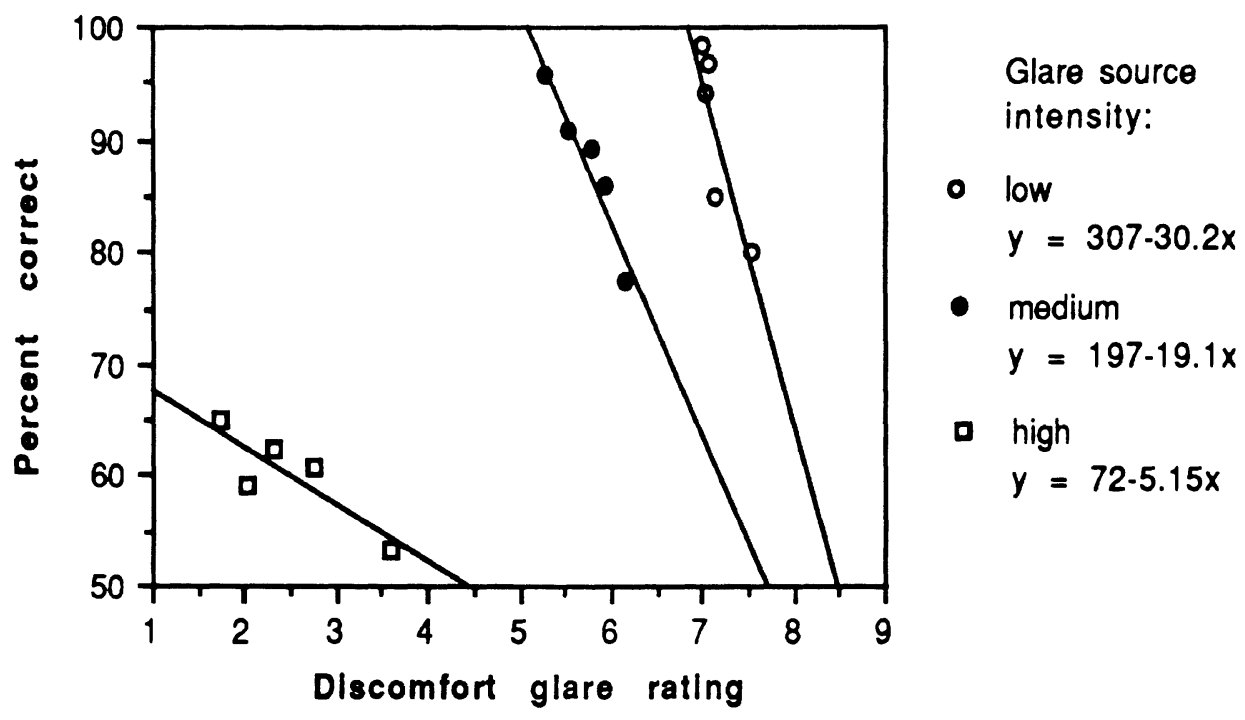

Figure 14. The tradeoff between percent correct and discomfort produced by changing mirror reflectivity, shown for each level of glare source intensity. The equations are for the regression lines shown. 
Discomfort glare ratings and confidence. In the above discussion of discomfort glare ratings we pointed out that those ratings are reasonably well predicted by the Schmidt-Clausen and Bindels model, but that there appeared to be systematic deviations from the model. Specifically, the slopes of the lines relating discomfort ratings to illumination at the eye point were not as steep as predicted in the low and medium glare source intensity conditions. One explanation for this is the effect of task difficulty on discomfort glare ratings. The effect of a glare source on comfort, discomfort glare, and its effect on performance of a visual task, disability glare, have often been regarded as independent effects. However, manipulations of task difficulty that have no effect on the illumination at the eye nevertheless have substantial effects on subjects' ratings of discomfort glare (Sivak et al., 1989). Those findings suggest that manipulations of mirror reflectivity in the present experiment may have had two opposed effects on discomfort ratings. Lowering reflectivity reduced illumination at the eye (which should have resulted in milder discomfort ratings), but it also increased the difficulty of the gap-location task (which should have resulted in more severe discomfort ratings). The effects of mirror reflectivity on discomfort ratings that can be seen in Figure 11 may result from a combination of these two influences. If so, measurements of task difficulty, or more importantly subjective task difficulty, may prove useful in accounting for discomfort ratings. This possibility is especially interesting in light of the interaction between mirror reflectivity and glare source intensity on confidence ratings. If confidence ratings are an indicator of subjective task difficulty, then the data in Figure 11 indicate that lower mirror reflectivity resulted in greatly increased subjective task difficulty when glare source intensity was low or medium, but virtually no change in subjective task difficulty when when glare source intensity was high. The most intense glare source may have resulted in levels of difficulty that were all too high for the subjects to discriminate among.

The above considerations suggest that the interaction of mirror reflectivity and glare source intensity on confidence ratings can be used to explain the interaction of those same two variables on discomfort ratings by taking into account the effect of subjective task difficulty on discomfort ratings. Figure 15 shows the same view of discomfort rating data as Figure 11, but with the predictions of two new models. The first model is simply a linear regression of discomfort ratings on the logarithm of lux values, and its prediction is shown by the straight line in Figure 15. The fit is good, $r^{2}=.87, t(13)=9.42, p<.001$. The agreement of this model with the Schmidt-Clausen and Bindels model is very good, as can be seen by comparison with Figure 11. This is not surprising in that Schmidt-Clausen and Bindels also predict discomfort ratings will be linear with the logarithm of lux at the eye. Their fit is not 
quite as good because they also specify the slope and intercept for the relationship, but their model comes admirably close to the best fitting line.

The second model whose predictions are shown in Figure 15 is a linear regression using as predictors both the log lux values and confidence ratings. Its predictions are shown as open squares. The fit is much improved over the model involving only log lux values. The proportion of variance accounted for is now $r^{2}=.97$, and the improvement in fit due to including confidence ratings is highly significant, $F(1,12)=44.16, p<.001$. Taking into account subjective task difficulty, as quantified by confidence ratings, seems to provide a good account of the relationships between mirror reflectivity, glare source intensity, and discomfort ratings. When glare source intensity was low or medium, the slopes of the lines relating discomfort ratings to reflectivity were not as steep as the Schmidt-Clausen and Bindels model predicts, because the increase in glare caused by the higher lux values associated with higher reflectivity was partially offset by the subjects' improved ability to see with higher reflectivity. When glare source intensity was high, subjective difficulty was always at a maximum and so did not change with mirror reflectivity. Mirror reflectivity in this condition therefore affected discomfort ratings only through increased illuminance, resulting in a steeper slope in the discomfort-reflectivity relationship, more in line with the Schmidt-Clausen and Bindels predictions.

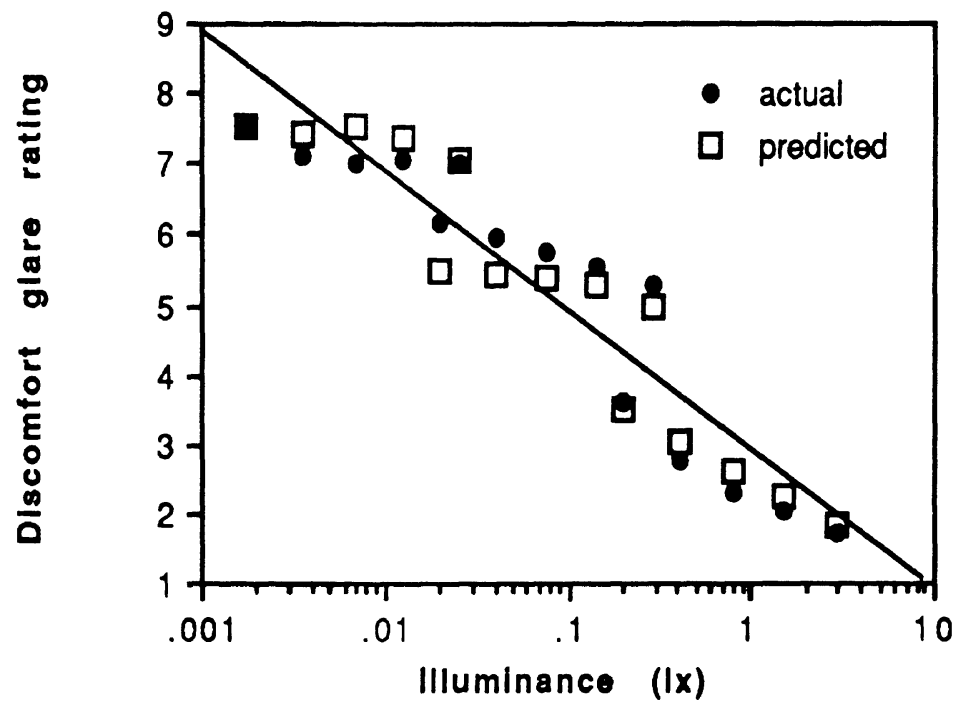

Figure 15. Discomfort glare ratings as a function of illumination at the subject's eye point, showing the fits of the best linear model using only lux value (straight line) and of the model using lux value and confidence rating (open squares). 


\section{CONCLUSIONS}

We began the present study with the belief that the problem of specifying rearview mirror reflectivity under nighttime glare conditions was one of assessing tradeoffs among rearward visibility, forward visibility, and discomfort glare. How should our understanding of the problem be influenced by the present results? First, although no single study can fully settle the issue of how all important performance measures vary with mirror reflectivity, this study offers support for the possibility that optimum reflectivity will be determined by an essentially linear tradeoff. Both gap-location performance and discomfort glare ratings seem to be well described as linear functions of log reflectivity within reasonable ranges of reflectivity. For the performance measure, there were some effects of age. However, in spite of a difference in overall performance, and some interaction effects that can be attributed to it, the effect of reflectivity on performance is similar across ages. Any strong nonlinearities or discontinuities in the functions relating performance to reflectivity would suggest that there is no reason to provide for more than a small number of discrete reflectivity levels. However, these results suggest that the continuous reflectivity control allowed by electrochromic mirrors causes continuous perceptual effects, and thereby can provide meaningful improvement in the control of the tradeoffs involved in night driving.

A second conclusion from this study is that subjects' awareness of their seeing ability, and specifically of how that ability is affected by mirror reflectivity in a situation in which reflectivity is varied automatically and without explicit notice, is conservative but otherwise in reasonable agreement with reality. This result is encouraging in that it suggests drivers cope well with the automatic, graded changes in reflectivity that an electrochromic mirror can

provide. But this conclusion should be limited to the type of visual task used here, a discrimination task. It is useful to distinguish such tasks from a second major class of tasks commonly recognized in the study of visual performance: detection tasks. In discrimination tasks an observer is required to decide which of a set of possible stimuli was presented, as here subjects were required to decide on each trial whether a gap-up or gap-down stimulus appeared. In detection tasks the observer must decide whether something or nothing appeared on a trial. Both types of task seem relevant to rearview mirror performance. Deciding which lane a distant but approaching car occupies is an example of a discrimination task that might be performed in a rearview mirror. Deciding whether the dirty and unlighted rear corner of another vehicle is just behind and in an adjacent lane before making a lane change is an example of a particularly difficult nighttime detection task that also might be performed in a rearview mirror. How well subjects are aware of the level of impairment they might experience in detection tasks as mirror reflectivity changes is an important additional question. 
It is interesting that subjects in this study consistently underestimated how well they were performing the gap-location task. If this represents a true misunderstanding of their abilities, it might have major consequences for the selection of rearview mirror reflectivity. For example, if a mirror were designed to provide adequate rearward visibility, but users underestimated how well they could see to the rear, it is possible that users would not accept the mirror even though the seeing ability it provides might be optimal. On the other hand, it may be that subjects' underestimation merely represents how they understand the confidence scale. Comparisons of subjects' estimates across conditions might be valid, but their overall use of the scale might be miscalibrated. Further work to explore these possibilities could prove valuable.

Finally, it is clear that subjects' ratings of discomfort glare are related to the difficulty of the concurrent visual task. The theoretical importance of this finding is that it calls into question the view that discomfort glare can be predicted directly from the illumination conditions (adaptation luminance, illumination at the eye, and glare angle) as embodied in the Schmidt-Clausen and Bindels model. The immediate practical significance of the finding is that discomfort glare must be measured under at least approximately the actual conditions of concern. For example, the slope for discomfort rating as a function of illumination predicted by the Schmidt-Clausen and Bindels model appears to be wrong when the variation in illumination is achieved by changes in mirror reflectivity, which also affects seeing ability.

\section{Future directions for research}

The ultimate goal of work on rearview mirror reflectivity should be the identification, quantification, and weighting of a set of measures appropriate for rating rearview mirror performance, with the understanding that a determination of the best reflectivity level for any situation involving glare from the rear will involve a tradeoff among several factors. This study has been devoted primarily to the first part of that problem: identifying appropriate measures and considering the problems of quantification in a preliminary way. Perhaps the most immediate way in which the present results should be extended is in a further effort to identify important measures of performance, specifically by investigating the effects of reflectivity on a detection task. Detection tasks are important in that they are a significant fraction of the visual tasks for which rearview mirrors are used. They are particularly interesting in that, unlike discrimination tasks, they involve the possibility that reduced visibility will bias observers toward one response, i.e. the decision that no stimulus is present. It is important that drivers not confuse the inability to see something, such as a vehicle in an adjacent lane, with the experience of seeing that nothing is there. Such confusions cannot arise with discrimination tasks, such as deciding which of two lanes an overtaking vehicle is in. 


\section{REFERENCES}

Bhise, V. D., Swigart, T. F., \& Farber, E. I. (1975). Development of a headlamp dimming request prediction model. In, Human Factors in Our Expanding Technology. Proceedings of the Human Factors Society 19th Annual Meeting. Santa Monica: Human Factors Society.

de Boer, J. B. (1967). Visual perception in road traffic and the field of vision of the motorist. In J. B. de Boer (Ed.), Public lighting (pp. 11-96). Eindhoven, The Netherlands: Philips Technical Library.

Flannagan, M. (1988). Human performance aspects of rearview mirrors: An appliedliterature review (Report No. UMTRI-88-20). Ann Arbor, MI: The University of Michigan Transportation Research Institute.

Flannagan, M., \& Sivak, M. (1990). Nighttime effectiveness of rearview mirrors: Driver attitudes and behaviors (SAE Technical Paper Series \#900567). Warrendale, Pennsylvania: Society of Automotive Engineers.

Helder, D. J. (1987). Design parameters for an automotive interior mirror (SAE Technical Paper Series \#870635). Warrendale, Pennsylvania: Society of Automotive Engineers.

Lynam, N. R. (1987). Electrochromic automotive day/night mirrors (SAE Technical Paper Series \#870636). Warrendale, Pennsylvania: Society of Automotive Engineers.

Olson, P. L., Jorgeson, C. M., \& Mortimer, R. G. (1974). Effects of rearview mirror reflectivity on drivers' comfort and performance (Report No. UM-HSRI-HF-74-22). Ann Arbor, MI: The University of Michigan Highway Safety Research Institute.

Olson, P. L., \& Sivak, M. (1984). Glare from automobile rear-vision mirrors. Human Factors, 26, 269-282.

Schmidt-Clausen, H.-J., \& Bindels, J. Th. H. (1974). Assessment of discomfort glare in motor vehicle lighting. Lighting Research and Technology, 6, 79-88.

Sivak, M., Flannagan, M, Ensing, M, \& Simmons, C. J. (1989). Discomfort glare is task dependent (Report No. UMTRI-89-27). Ann Arbor, MI: The University of Michigan Transportation Research Institute. 